\title{
A Cognitive Study on Linguistic Expressions of Spatial Metaphors
}

\author{
He Pan \\ Tianjin University Renai College \\ Tianjin, China
}

\begin{abstract}
Metaphor has long been studied both in the western world and in China. From the viewpoint of the contemporary metaphorical theory, many of our fundamental concepts are structured through spatial metaphors rooted in our physical, social and cultural experiences. Cognitive linguists view knowledge, including linguistic knowledge, as arising out of people's interaction with the world, and among this knowledge, the primacy is given to space. The reason is that spatial relations are relatively concrete as they are the relationships that are most direct to us human beings and thus most easily accessible. Space serves as a fundamental conceptual structuring device in language. It is served as a category with which human beings interact with their environment. Meanwhile, spatial terms are often used in fundamentally non-spatial domains, through metaphorical mapping, lending a spatial structure to the non-spatial domains. This paper focuses on the linguistic expressions of spatial metaphors from a cognitive approach.
\end{abstract}

Keywords-spatial metaphor; cognitive linguistics; conceptual structure

\section{INTRODUCTION}

From the perspective of cognitive linguistics, abstract concepts are at least in part understood and expressed metaphorically in spatial terms and that abstract reason is achieved by using certain mechanisms for the perception of spatial relations. Metaphor projects the image-schematic structure of the source domain onto the target domain in a way that is consistent with inherent target domain structure. It casts the abstract and the nonphysical into the concrete and the physical, usually with spatial dimensions. Indeed, most image-schemas, such as Source-Path-Goal, Object, Containment, Balance, Links-, and Cycle are spatial in nature. Even various invisible force schema, such as Compulsion, Attraction, Counterforce, Diversion (Johnson 1987), bring about spatial consequences.

Most of the conceptual structure of a natural language is metaphorical in nature. The conceptual structure is grounded in physical and cultural experience, as are the conventional metaphors. Meaning, therefore, is never disembodied or objective and is always grounded in the acquisition and use of a conceptual system. According to cognitive linguists, the formation of concept is rooted in common bodily experience, especially spatial experiences, which constrain people's metaphorical construction to the abstract concepts. Spatial relations play a basic role in people's cognition process.
Through human beings' active cognition activities, the spatial relations in the objective world form conceptualized spatial relations. When the relations are reflected in language, they become spatial relations in language.

\section{RESEARCH BACKGROUND}

Metaphor has long been studied in the western world, and it is a heated topic in the western academic field ever since 2000 years ago. Scholars like Aristotle, Quintilian, Richards, Black, and Lakoff, Johnson, and many others have contributed a lot to the metaphorical study. The definition, mechanism of function and extension of usage of metaphor have all been focus questions discussed by the above scholars. Even though it has not reached a unanimous agreement, there is no doubt that metaphor is a problem worth to be further researched. The systemic study of metaphor in the western world can be traced back to Aristotle. In his classic works Poetics and Rhetoric, he referred to the formation method and rhetoric function of metaphor for many times. Since Aristotle, metaphor studies have developed in the following line:

- From Aristotle to 1930 s, a rhetoric study period, metaphor is considered as a deviation from the ordinary language or a figure of speech, and an ornamental device in the eyes of the rhetoricians and literary critics, who regard that the metaphor study is restricted to poetics and stylistics.

- From 1930s to 1970 s, a semantic study period, Richards put forward the interactive view about metaphor. He and his followers begin to notice the cognitive force of metaphor. Until then, metaphor gets rid of its fate being as the deviation of language.

- Until the late 1970s, the landmark work Metaphors We Live By written by Lakoff and Johnson announced that the study of metaphor has since stepped to a new stage that is to say, studying metaphor from a cognitive point of view. In the eyes of cognitive linguists, metaphor is ubiquitous in our everyday language "metaphor is pervasive in everyday life, not just in language but in thought and action. Our ordinary conceptual system, in terms of which we both think and act, is fundamentally metaphorical in nature" (Lakoff, 1980:3) 
The metaphor defined by Lakoff above is called conceptual metaphor, which in certain cultures becomes a systemic consistent whole, named metaphorical concept system playing a major and decisive role in people's cognition to the objective world. For the cognitive linguists, metaphor is a property of concepts and it is defined as understanding one conceptual domain in terms of another. The function of metaphor is to understand the abstract concepts through more concrete ones which they are quite familiar with. In the Conceptual Metaphor Theory, Lakoff and Johnson (1980:5) claim that "the essence of metaphor is understanding and experiencing one kind of thing in terms of another". In Metaphors We Live By, Lakoff classified metaphor into three kinds: structural metaphor, orientational metaphor and ontological metaphor.

Among the three kinds of metaphors above, orientational metaphor or spatial metaphor is the central concern of the present study. Orientational metaphor refers to a series of metaphorical concepts constructed according to spatial orientation. Spatial orientations come from the interaction between people and the nature. They are the most basic concepts that people live on: up-down, front-back, deepshallow, and central-peripheral. People map those concrete concepts to abstract concepts like emotion, physical conditions, quantity, social status, for example: Happy is up; sad is down. Health and life are up; sickness and death are down. More is up; less is down. Having Control Or Force Is Up; Being Subject To Control Is Down. The major questions which the author believes to be worth exploring will be presented in the following section.

Different from studies on metaphor in the west, there are no systemic theories about metaphor in China. Metaphor has long been discussed as a figure of speech in the rhetoric field and it is considered as a hyponym of its superordinate: biyu, which consists of mingyu (simile), anyu (metaphor) and jieyu (loan metaphor-describing the vehicle directly with no mention of the tenor). In China, the status of metaphor as a figure of speech didn't change until 1980s. From then on, many scholars began to introduce metaphor theories from the west especially conceptual metaphor theory proposed by Lakoff. Since then, metaphor becomes a question individually discussed as a cognitive instrument. In the following section, biyu studies in Chinese rhetoric field and cognitive studies on metaphor in contemporary and current China will be illustrated.

\section{THEORETICAL FRAMEWORK}

The present study is built on cognitive metaphor theory, claiming that spatial perception is pre-linguistic and taking the possible existence of a universal spatial system as a hypothesis. By metaphor, spatial representations are used for more abstract concepts. To explain this idea further we next have to outline the cognitive linguistics theory.

\section{A. Cognitive Linguistics}

Cognitive linguistics refers to the new linguistic school which was born in the late 1970 s and got a rapid development in the 1980s and 1990s, and became an important researching field. The representatives in cognitive linguistic research are G. Lakoff, R. Langacker, C. Fillmore, M. Johnson, J. R. Tayor, D. Geeraerts, P. Kay, G. Fauconnier and L. Talmy. Important studies in cognitive linguistics include Len Talmy's work on figure and ground, Ronald Langacker's cognitive grammar framework, George Lakoff's research on metaphor, gestalts, categories and prototypes, Fillmore's frame semantics, and Fauconnier's mental spaces. Beside the above achievements, there are still hundreds of scholars who are engaged in the deeper research in this field, making a huge number of published researches on the theories and their applications.

Since cognitive linguistics has not formed a complete theoretical system yet, different scholars have different views toward it. "Cognitive linguistics' ultimate goal is to understand how human cognition motivates the phenomena of language, to be described in terms of abstract trends rather than air-tight, absolute rules." (Laura Janda, 2000: 5) In order to achieve the researching goal, cognitive linguistics mainly focuses its concerns on the following aspects: prototypes and categories, levels of categorization, conceptual metaphors and metonymies, figure and ground, iconicity, grammaticalization and lexical change. Metaphor plays an important role in cognitive linguistics, and Lakoff is one of the prominent cognitivists, who have done great contribution to metaphor studies.

\section{B. Image Schema}

Taking an experiential view, Lakoff and Johnson argue that a small number of schemas of physical-world relations, which they call "image schemas", underlie the metaphorical structure of everyday language, and are derived from our fundamental experiences of being embodied in the world.

The definition of image schema has different expressions but their central meaning is the same. Human beings have bodies and we are in kinds of activities in anywhere and at any time. When we observe the surroundings and walk, eat or do other activities, our bodies are in constant contact and interaction with the outside world. Image schema comes into beings from such activities which seem to be irrelevant with each other, and makes such activities consistent and gives abstract activities concrete structures. Therefore, we can use image schemas to understand such activities and make the seemingly irrelevant activities relevant.

In their path-breaking 1980 publication, George Lakoff and Mark Johnson jointly introduced the notion of 'image schema' as one of experientialism's major foundational pillars, though with the linguist's and philosopher's different sources of inspiration and foci of interest. According to Johnson, an image schema is a mental pattern that recurrently provides structured understanding of various experiences, and is available for use in metaphor as a source domain to provide an understanding of yet other experiences. Image schemas to Lakoff and Johnson are like CONTAINER, PATHS, LINKS, FORCES, BALANCE, and in various orientations and relations, like IN-OUT, UPDOWN, FRONT-BACK, CENTER-PERIPHERY, PARTWHOLE. Image schemas are directly meaningful because 
they have been repeatedly in function in our lives. It is not a concrete image, but an abstract cognitive structure in the human cognitive system. (Zhao Yanfang, 2001) Ungerer and Schmid elaborate that an image schema is not just an abstract semantic principle, but should be understood as a mental picture that is more elementary than both concrete categories and abstract principles (Ungerer \& Schmid, 2001).

\section{Metaphor Mapping}

According to Lakoff (1987: 276), each metaphor has a source domain (relatively clearly structured), a target domain (relatively less clearly structured), and a source-to-target mapping. A metaphor is a mapping from a source domain to a target domain. In other words, whenever a person takes a concept that has been formed in one domain and tries to implement it in another, a metaphor has occurred. The domain in which virtually all human knowledge is formed is that of a human body in physical space, which usually serves as the source domain for metaphor. Common target domains are time, emotions, and states of being. Metaphor is a very robust phenomenon for all language. It is quite impossible to speak any language without mastering the metaphorical conventions embedded in it.

As metaphors are "pervasive in everyday life, not just in language but in thought and action" (Lakoff \& Johnson 1980: 3) as well, they are used permanently in everyday communication, politics, education, science etc. Metaphorical mappings are used to help us comprehend most universal and basic concepts of the world we live in. Such basic concepts derive from our concrete daily experiences and our knowledge of the world and then are projected onto abstract concepts, such as time, state and quality. For example, the conceptual metaphor MORE IS UP (Lakoff \& Johnson 1980: 23) mirrors a mapping process, in which vertical movement is projected onto quantity.

As part of cognitive and metaphorical processes, such mappings arise more or less automatically and unconsciously, and thus affect the way we experience, think and interact within our environment. For example, the correspondence between the domains ARGUMENT and WAR "arises from a correlation in our normal everyday experiences." (Lakoff \& Johnson 1999: 47) This shared understanding influences and reflects our interpretation of reality. Through the conceptual metaphor ARGUMENT IS WAR, we can actually win or lose arguments, e.g. I've never won an argument with him. We see the person we are arguing with as an opponent and we plan and use strategies, e.g. If you use that strategy, he'll wipe you out. We attack our opponent's positions and we defend our own, e.g. I attacked every weak point in his argument. Many of the things we do in arguing are partially structured by the concept of war.

Metaphor mapping is usually highly selective. "Mappings are asymmetric and partial." (Lakoff 1993: 35) It is by no means a one-to-one mapping of all the information from a source domain to a target domain. For example, the fact that in English fire is used as a source domain for understanding anger. Examples like His temper is like a powder-keg; She's white-hot with rage; I'm fuming, doing a slow burn, etc. (Lakoff 1987: 380-414) do not mean we expect anger to be something we can light with a match, use for cooking, or use to produce ashes. Metaphor is motivated by relevant information that is salient in human experience; it highlights some facts about the target domain, but hides others. The behavior of metaphor is likewise well-motivated but not entirely predictable. (Laura 2000: 12-13)

Knowledge in the source domain gets mapped onto knowledge in the target domain. Our knowledge of a domain allows us to draw inferences about that domain. When a domain serves as a source domain for metaphoric mapping, inference patterns in the source domain are mapped on the target domain. For example, if you hit dead end, you cannot go on in the same direction and have to find another route. If you hit a metaphorical dead end in life you must find another course of action.

\section{Spatial Metaphor Studies}

Cognitive linguists view knowledge, including linguistic knowledge, as arising out of people's interaction with the world, and among this knowledge, the primacy is given to space. The reason is that spatial relations are relatively concrete as they are the relationships that are most direct to us human beings and thus most easily accessible. Space serves as a fundamental conceptual structuring device in language. It is served as a category with which human beings interact with their environment. Meanwhile, spatial terms are often used in fundamentally non-spatial domains, through metaphorical mapping, lending a spatial structure to the nonspatial domains.

\section{A. Definition of Spatial Metaphor}

Spatial metaphor refers to the metaphor with mapping from spatial orientation as source domain to non-spatial domain (usually abstract domain) as target domain. (Lakoff 1980: 27) Cognitive linguists generally admit that spatial metaphor plays a significant role in conceptualization of human being, because most of abstract things are understood and expressed in terms of spatial metaphors. Spatial metaphors have close relationship with spatial orientation: up-down; in-out; front-back; on-off; deep-shallow; centralperipheral. These spatial orientations come into being from the interaction between human being and nature and they serve as the most basic bodily experience for mankind. People project spatial concepts onto abstract concepts such as time, emotion, physical state, quantity and social status, so the expressions of abstract concepts in terms of spatial concepts are gradually formed. For example, the conceptual metaphor HAPPY IS UP gives the concept HAPPY a spatial orientation UP. Corresponding metaphorical expression is like I'm feeling up today. That boosted my spirits. My spirits rose. You're in high spirits.

\section{B. Nature of Spatial Metaphor}

In previous part, we have illustrated spatial metaphor studies by Lakoff \& Johnson (1980: 14-17). To be more specific, the author classified the above metaphorical concepts into four categories, that is to say: quantity, social 
status, time and state. In order to be simple and concise, examples and physical and cultural basis are deleted.

- Spatial domain mapped onto domain of quantity

More is up; less is down

- Spatial domain mapped onto the domain of social status

Having control or force is up; being subject to control or force is down

High status is up; low status is down

- Spatial domain mapped onto the domain of time

Foreseeable future events are up (and ahead)

- Spatial domain mapped onto the domain of the state of human beings

Happy is up; sad is down

Consicious is up; unconscious is down

Health and life are up; sickness and death are down

Good is up; bad is down

Virtue is up; depravity is down

Rational is up; emotional is down

In the mapping processes from spatial domain to other cognitive domains, things changed are conceptual categories and what unchanged are their relations which are realized as image schemas in most situations. Those image schemas expressing spatial relations become the grounding for us to comprehend their internal correlations. "Image schema is a powerful productive cognitive schema. People used to project spatial categories and relations onto non-spatial categories and relations in order to comprehend more abstract concepts.” (Li Yuming, 1999: 64)

\section{Characteristics of Spatial Metaphor}

Lakoff and Johnson (1980: 17-19) notice the following characteristics of spatial metaphors:

- Most of our fundamental concepts are organized in terms of one or more spatial metaphors.

- There is an internal systematicity to each spatial metaphor.

- There is an overall external systematicity among the various spatial metaphors, which defines coherence among them.

- Spatial metaphors are rooted in physical and cultural experience; they are not randomly assigned.

- In many cases spatialization is so essential a part of a concept that it is difficult for us to imagine any alternative metaphor that might structure the concept.

Our physical and cultural experience provides many possible bases for spatial metaphors. Which ones are chosen, and which ones become salient, may vary from culture to culture.

\section{Spatial Metaphor Studies in Western Countries}

For spatial metaphors, Lakoff and Johnson (1980) have talked more by using English expressions. They consider that human beings build up a great number of spatial concepts: such as up/down, high/low, front/back, large/small etc. they claim that spatial terms are metaphorically extended to other concepts such as quantity, time, social hierarchy, emotion...

In Lakoff \& Johnson (1980: 14-17), illustrations of spatial metaphors in terms of Up-down and the physical and cultural experience that the metaphorical concept Up-down arisen from are explained in the following:

\section{Happy is up; sad is down}

E.g. I'm feeling up. That boosted my spirits. You're in high spirits. Thinking about her always gives lift. I'm feeling down. I'm depressed. I fell into a depression. My spirits sank.

Physical basis: Drooping posture typically goes along with sadness and depression, erect posture with a positive emotional state.

Consicious is up; unconscious is down

E.g. Get up. Wake up. I'm up today. He fell asleep. He dropped off to sleep. He sank into a coma.

Physical basis: Humans and most other mammals sleep lying down and stand up when they awaken respectively.

Health and life are up; sickness and death are down

E.g. He's in the peak of health. As to his health, he's way up there. He's sinking fast. He came down with the flu. He dropped dead.

Physical basis: Serious illness forces us to lie down physically. When you're dead, you are physically down.

Having control or force is up; being subject to control or force is down

E.g. I have control over her. I am on top of the situation. He's at the height of his power. His power is on the decline.

Physical basis: physical size typically correlates with physical strength, and the victor in a fight is typically on top.

More is up; less is down

E.g. The number of books printed each year keeps going up. My income rose last year. The number of errors he made is incredibly low. If you are too hot, turn the heat down.

Physical basis: if you add more of a substance or of physical objects to a container or pile, the level goes up.

Foreseeable future events are up (and ahead)

E.g. All upcoming events are listed in the paper. I'm afraid of what's up ahead of us.

Physical basis: normally our eyes look in the direction in which we typically move (ahead, forward). As an object approaches a person (or the person approaches the object), 
the object appears larger. Since the ground is perceived as being fixed, the top of the object appears to be moving upward in the person's field of vision.

\section{High status is up; low status is down}

E.g. She'll rise to the top. He's at the peak of his career. He's at the bottom of the social hierarchy.

Social and physical basis: status is correlated with (social) power and (physical) power is UP.

\section{Good is up; bad is down}

E.g. Things are looking up. He does high-quality work. Things are at an all-time low.

Physical basis for personal well-being: happiness, health, life, and control-the things that principally characterize what is good for a person-are all UP.

\section{Virtue is up; depravity is down}

E.g. He is high minded. She has high standards. She is upright. That was a low trick. He fell into the abyss of depravity.

Physical and social basis: good is up for a person (physical basis), together with a metaphor that we will discuss below, society is a person (in the version where you are not identifying with your society). To be virtuous is to act in accordance with the standards set by the society/person to maintain its well-being. Virtue is up because virtuous actions correlate with social well-being from the society/person's point of view. Since socially based metaphors are part of the culture, it's the society/person's point of view that counts.

\section{Rational is up; emotional is down}

E.g. The discussion fell to the emotional level, but I raised it back up to the rational plane. He couldn't rise above his emotions.

Physical and cultural basis: in our culture people view themselves as being in control over animals, plants, and their physical environment, and it is their unique ability to reason that places human beings above other animals and gives them this control. Control is up thus provides a basis for man is up and therefore rational is up.

Through the above illustration, Lakoff and Johnson clearly pose spatial experience as primary source for such basic concepts. Such a view is backed by Levinson's observation that "spatial cognition is at the heart of our thinking. It has long been noted that spatial thinking provides us with analogies and tools for understanding other domains. The pervasive spatial metaphors of everyday language, the evocativeness of place in memory...spatial cognition probably plays this central role because it seems to be the evolutionarily earliest domain of systematic cross-modal cognition: any animal needs to relate what its eyes, ears and limbs tell it about the immediate structure of the world around it." (Levinson 2003: p.xvii)

To put it in the words of Lakoff and Johnson (1980: p.59), "we typically conceptualize the nonphysical in terms of the physical-that is, we conceptualize the less clearly delineated in terms of the more clearly delineated". According to this position, space is the physical domain par excellence, and other physical domains (such as time), as well as nonphysical ones (such as emotions), are conceptualized in terms of it via metaphors e.g. John is in love, The affair is out of control, I am up in the air. It is then clear that basic concepts, being themselves understood independent of any metaphorical structure, provide the ultimate basis for grounding non-basic, metaphorically understood concepts in our daily experience.

\section{E. Spatial Metaphor Studies in China}

In the past years, spatial metaphor studies have been one of the most active research subjects in cognitive linguistic field. In 1997, Qi Huyang wrote Studies on Modern Chinese Spatial Problems and in 1998, Chu Zexiang wrote Systematic Studies on Modern Chinese Space and both of them studied the panorama of the space system in modern Chinese; however, they haven't paid much attention on spatial metaphors in Chinese. In On Basic Strategies of the Cognitive Progress of Chinese Spatial Position, Fang Jingmin pointed out spatial positions reflected the cognitive progress of the language society to the spatial relations in the cognitive space. It differs from the common linear, tangible and superficial syntactic structure but a dimensional, abstract and deep cognitive structure.

In 1999, Lan Chun published A Cognitive Approach to Spatial Metaphors in English and Chinese. In 2005, she further published Cognitive Linguistics and Metaphor Studies. In those two books, she made both qualitative and quantitative studies to shang/up expressions and xia/down expressions in selected from real Chinese and English languages and then made conclusions that the correspondence between the metaphorical extensions of SHANG and XIA on the one hand, and of UP and DOWN on the other, is remarkable. This correspondence offers support to the possible existence of a universal spatial metaphor system.

Scholars in China also discussed English spatial metaphors for example Zhao Yingling demonstrated characteristics of spatial metaphors like non-arbitrary, generative, systematic and multi-dimensional through real data analysis to verticality metaphor based on rhetoric and linguistic theories in Characteristic Analysis to English Spatial Metaphors. In On Spatial and Metaphorical Sense Cognition of up, Tao Wenhao discussed the spatial meaning, cognitive process and metaphoric extensions.

On the aspect of contrastive study on spatial words, most scholars tend to study them related to culture. In Contrastive Study of Spatial Metaphors between Russian and Chinese, Zhang Feng made contrastive analysis of metaphorical concepts of shang-xia in Chinese and Russian and the author pointed out the metaphorical concepts of shang-xia in time, quantity and social status. In Social Status in English and Chinese Spatial Words, Wu Xinhua and Zhao Xiong discussed social status reflected in spatial words by using groups of common spatial words such as "shang-xia (updown), zио-уоu (left-right), dong-xi (east-west) and nan-bei 
(south- north)". Henceforth, more and more Chinese linguists become interested in spatial metaphor studies and make great contributions to the study.

\section{F. Cognitive Model of Spatial Metaphor}

As demonstrated in the previous sections, cognitive linguists claim that metaphor is to understand one domain of experience in terms of another. This point shows that our understanding to the outside world takes place in terms of the entire domains of experience which come from our interaction with the physical and cultural environment. Therefore, metaphor and culture are related with each other. Spatial metaphors, as one kind of metaphors, cannot be get rid of the influence by culture.

The application of metaphorical concept shows that the metaphor cognition is universal across cultures, but at the same time, it has some differences in their application because of their different social and cultural backgrounds.

\section{G. Universalities Between English and Chinese Spatial Metaphors}

Metaphor cannot be studied separately from culture. In Metaphor We Live by, Lakoff and Johnson (1980) proposed that metaphor does not occur primarily in language but in thought. In a fundamental sense, human cognition is embodied. "Experiential realism characterizes meaning in terms of embodiment, that is, in terms of our collective biological capacities and our physical and social experience as beings functioning in our environment" (Lakoff 1987: 266-267). Therefore, there are universalities among human being's cognition, which drive from people's daily bodily experience. Spatial concepts are formed during the period of infant. In the process of language input, the forms to express orientations are easy to understand, including a large number of spatial metaphors, which little by little create the formation of thinking patterns. As for this, a great cognitive similarity is shared by different people. There are many similar spatial metaphors used both in English and Chinese to express the same abstract thoughts. In Chinese, there are also some examples which can be shown as counterparts of their English corresponding expressions. E.g.

I'm feeling up.

$$
\text { 我感觉很高兴。 }
$$

The number of books printed

each year keeps going up.

书籍的印刷数量逐年上升。

He’s at the peak of his career. 他处于事业的最高峰。

\section{H. Differences Between English and Chinese Spatial Metaphors}

Metaphor is assumed as a kind of cognitive pattern to objective world as well as the reflection of culture. In different cultures, the standard practice of metaphorical concept show the universality of metaphorical cognition and the cognitive generality of different nationalities, on the other hand, there are some certain cultural differences in metaphorical concept due to the influence of different cultures. For example, when the Chinese Shang is projected into the domain of time, the conceptual metaphor is toward an earlier time is Shang, while the conceptual metaphor of the English up is toward a latter time is up. We cannot understand metaphor without its social and cultural background, so it is of great importance to master the social and cultural knowledge of the target language in the study of metaphor.

\section{CONCLUSION}

From the above statement of previous studies on spatial metaphors, a prediction can be referred that thing about the nature of our bodies and brains, and then considering the kinds of physical and cultural interactions we participate in due to the kinds of interest and purposes we have, there may well be universal image schemas, metaphorical concepts, or cognitive structures. The conclusion remains tentative at the stage because the cross-linguistic and cross-cultural studies that could identify possible empirical universals have simply not been carried out extensively enough at the present time. The investigation of the metaphorical extensions of spatial metaphor theoretically supports Lakoff's conceptual metaphor theory. Metaphor is a way to investigate human mind through language analysis. Metaphor is one of the main mechanisms which enable us to understand abstract concepts and to carry out abstract reasoning. In addition, the present study provides evidences for some cognitive linguists' prediction that there may indeed exist a universal spatial metaphorical system. The study of cognitive linguistics and further the cognitive and cultural models of linguistic expression of metaphor especially to spatial metaphor lay a solid foundation to the thesis and will help a lot to the later cognitive analysis to spatial expressions. In application, this research is helpful for language teaching and learning because metaphorical theory has positive guiding effect and applicable value for language teaching and learning. Language teachers can use metaphorical theory to explain the changing and developing process of language meaning and interactive relations among lexical words.

\section{REFERENCES}

[1] Johnson, M. The Body in the mind: The bodily basis of meaning, imagination, and reason. Chicago, IL : University of Chicago Press, 1987.

[2] Johnson, M. "Philosophical Implications of Cognitive Semantics". Cognitive Linguistics 3-4, pp. 345-66, 1992.

[3] Lakoff, G. \& Johnson, M. Metaphors We Live By. Chicago: The University of Chicago Press, 1980.

[4] Lakoff, G. Women, Fire, and Dangerous Things: What Categories Reveal about the Mind. [M]. Chicago: The University of Chicago Press, 1987.

[5] Lakoff, G. \& Turner, M. More Than Cool Reason---A Field Guide to Poetic Metaphor. Chicago: The University of Chicago Press, 1989.

[6] Lakoff, G. The Contemporary Theory of Metaphor. Cambridge: Cambridge University Press, 1993.

[7] Lakoff, G. "What is a Conceptual System?" In Willis F Overton and David S. Palermo (eds.), pp. 41-90, 1994.

[8] Lakoff, G. \& Mark Johnson. Philosophy in the Flesh---The Embodied Mind and its Challenge to Western Thought. New York: Basic Books, 1999. 
[9] Lan Chun. Cognitive Linguistics and Metaphor Studies. Beijing: Foreign Language Teaching and Research Press, 2005.

[10] Laura Janda. Cognitive Linguistics. SLING2K Position Paper. 2000.

[11] Levinson, S. C. Space in Language and Cognition: Explorations in Cognitive Diversity. Cambridge: Cambridge University Press, 2003.

[12] Pütz, Martin \& Rene Dirven, The Construal of Space in Language and Thought. Berlin: Mouton de Gruyter, 1996.

[13] Tyler, A. Applying Cognitive Linguistics to Learning the Semantics of English to, for and at: An Experimental Investigation[J]. Vigo International Journal of Applied Linguistics, vol. 8, pp. 181-205, 2011.

[14] Ungerer, F. \& Schmid, H. J. An Introduction to Cognitive Linguistics. Beijing: Foreign Language Teaching and Research Press, 2001

[15] Wang Yin. Cognitive Linguistics. Shanghai: Shanghai Foreign Language Education Press, 2007.

[16] Zhao Yanfang. An Introduction to Cognitive Linguistics. Shanghai: Shanghai Foreign Language Education Press, 2001. 